\title{
McGill
}

University

Montreal

\author{
Mario Bunge
}

\section{MATERIALISM TODAY}

Materialism is a family of ontofogies, or exfreme/y general doctrines about the world. What all the members of that family have in common is the thesis that everything that exists really is material — or, stated negatively, that immaterial objects such as ideas have no existence independent of material things such as brains. Aside from this common core, materialist ontologies may differ widely. It is only by adding further requirements that a definite materialist ontology will be individuated or built. We choose two: exactness and consistency with contemporary science. Let us peek at these conditions.

\section{EXACTNESS AND CONSISTENCY WITH CONTEMPORARY SCIENCE}

So far materialism has been a rather amorphous body of somewhat vague beliefs. How can one transform such a doctrine into a system of clearly stated hypotheses consistent with contemporary knowledge, in particular logic, mathematics, natural science, social science, and technology? In general, how can one attempt to overhaul a philosophy? The short answer is: By substituting exact formulas for vague metaphors, by weeding out the obsolete theses, and incorporating new hypotheses consistent with contemporary knowledge.

Let us deal with exactification first. It consists in replacing vagueness with precision. This goal is attained by using, wherever necessary, the exact and rich languages of logic and mathematics instead of ordinary language, which is incurably fuzzy and poor (cf. Bunge, Ed. 1972). But almost any philosophy, provided it is not irrationalistic, can be rendered precise and clear, i.e. can be reformulated with the help of logical and mathematical concepts. Hence formalization, though necessary for turning an unorganized body of vague 
theses into a hypothetico-deductive system, is insufficient for overhauling a philosophy.

When we say that philosophy $\mathrm{X}$ is obsolete we intend to state that $\mathrm{X}$ fails to meet contemporary standards of exactness or that $\mathrm{X}$ is at variance with contemporary substantive knowledge about the world and human experience. Materialism is a case in point, for it is not only inexact but has also failed to propose precise and up-todate answers to a number of central philosophical questions. However, there is a difference between materialism and other philosophies, namely that its main tenets, however imprecise, are by and large countenanced by contemporary science. Indeed, it can be argued that science investigates only material (or concrete) objects, and recognizes no immaterial ones - except for such objects as concepts, properties, and relations, none of which need be assumed to be self-existent.

Let us apply the exactness rule and the condition of consistency with science to the definition of the concept of matter.

\section{DEFINING MATTER}

The most popular definitions of the concept of matter offered in the past are inadequate. Material entities cannot be identified with massive objects, let alone solid ones, since the discovery of massless fields such as the electromagnetic and neutrino ones. And material objects cannot be defined as those which exist independently of the subject, because an objective idealist will assert the autonomous existence of immaterial objects such as ideas. In short, whereas the first definition has turned out to be scientifically obsolete, the second has always been philosophically inadequate. (For further inadequate definitions see Coraman 1971.)

We take our clue from contemporary science, according to which material objects, unlike ideal ones, are changeable. Even the so-called elementary particles are either unstable or, if long-lived, they change in various ways by virtue of their interactions with other entities (particles or fields). On the other hand a conceptual object, such as the number 3 or the Pythagorean theorem, is not supposed to be in any state, let alone to undergo changes of state. Thus it makes no sense to ask 'How is 3 doing today?' or 'What is the equation of motion (or the field equation or the transmutation schema) of the Pythagorean theorem?'

We may then characterize a material object as one that can be in at least two different states, so that it can jump from one to the other. (Actually even the simplest material entity, such as an electron or a photon, can be at a given time in any of infinitely many different states.) That is, if $\mathrm{x}$ is a material object and 
$S y(x)$ a state space for $x$, then the numerosity of the latter is at least 2 , and conversely.

It might be objected that disembodied souls, such as were posited by Plato and Descartes, and the ghosts said to haunt the Scottish castles, are changeable yet immaterial, so they prove the inadequacy of our definition. Not so, for this definition happens to belong to a materialist ontology, where there is no room for disembodied objects, and where mental states are brain states. Besides, no state spaces can be built to represent immaterial objects; this is why mentalist psychology has remained nonmathematical.

(We need not go here into the technique for building a state space $S y(x)$ for a thing $x$ relative to a reference frame $y$, for which see Bunge 1977 and 1979. Suffice it to say that it is a tacit epistemological postulate of contemporary science that, given any thing $x$ of which we know some properties, it is possible (a) to represent each property of $x$ by some mathematical function, and $(b)$ to collect all such functions into a single function, called the state function of $x$, which (c) is assumed to satisfy some law statement. Each value of that function represents a state of $x$ relative to the given reference frame $y$. The collection of such values, compatible with the laws of $x$, is called the nomological state space of $x$ relative to $y$. As time goes by the thing moves from one state to another, slowly relative to some frames, fast relative to others.)

In short, we shall adopt

Definition 1. An object $x$ is a material object (or entity) if, and only if, for every reference frame $y$, if $S y(x)$ is a state space for $x$, then $S y(x)$ contains at least two elements. Otherwise $x$ is an immaterial object (or nonentity).

Note that this is a set and thus a concept, not an entity: it is the collection of all

More briefly,

$\mu x=d f(y)$ (If $S_{y}(x)$ is a state space for $x$, then $\left.\left|S_{y}(x)\right| \geqslant 2\right)$.

This definition allows one to partition every set of objects into entities and nonentities. It also allows one to construct

Definition 2. Matter is (identical with) the set of all material objects.

In symbols,

$$
M=d f\{x \mid \mu x\} .
$$

past, present and future entities. (Or, if preferred, $M$ is the extension of the predicate ${ }^{\mu}$, $r$ read 'is material'.) Hence if we want to keep within materialism we cannot say that matter exists (except conceptually of course). We shall assume instead that individual material objects, and only they, exist. But this point calls for another section.

\section{THE CENTRAL POSTULATE OF MATERIALISM}

In order to state the central hypothesis of materialism we need not only the concept of matter but also that of reality for, according to materialism, all and only 
material objects are real. One way of defining the predicate, is real" is in terms of action or influence, namely thus. An object is real if, and only if, it influences, or is influenced by, another object, or is composed exclusively of real objects. (The second disjunct is needed to make room for the universe as a whole which, though unifluenced by anything else, is itself composed of real entities.) More precisely, we propose

Definition 3. An object $x$ is real if, and only if, either (a) there is at least another object $y$ whose states are (or would be) different in the absence of $x$, or $(b)$ every component of $x$ modifies the states of some other components of $x$.

Definition 4. Reality is the set of all real objects.

Note that, since ,reality” has been defined as a set, it is itself unreal, for sets are incapable of influencing anything. (There is nothing wrong with this, for wholes need not possess all the properties of their parts.) Note also the contrast between Definition 4 and the vulgar or eclectic notion of reality as the sum total of all objects, whether or not they can act upon or be acted upon by other objects. Finally note that we are not defining ,reality” as existence independent of the subject of knowledge, and this for two reasons. First because human creations do not come into being apart from us. (Thus a book, though real, owes its existence to its author and publisher.) Second because subjects too are supposed to be real.

We are now ready to state the hypothesis shared by all materialist ontologies:

Postulate 1. An object is real (or exists really) if, and only if, it is material. (Shorter: All and only material objects are real.)

This assumption bridges Definitions 1 and 3. By virtue of Definition 2 and 4, Postulate 1 is equivalent to: Reality is (identical with) matter. Put negatively: Immaterial objects (nonentities) are unreal. In particular the properties, relations, and changes in either, of material objects are real only in a derivative manner: strictly speaking they are abstractions. For example, the distances between entities are not real: only spaced things are. Likewise events are not real: what is real is the entire changing thing. (However, there is no harm in talking about the properties of entities, and their changes, as being real provided we do not detach them from the things themselves.)

A standard objection to materialism can now be answered. It is that space and time, though surely immaterial, must be reckoned with: for, are not things supposed to exist in (regions of) space and time? The materialist answer is the relational theory (or rather theories) of space and time adumbrated by the preceding remark. According to it spacetime, far from existing on its own, is the basic network of changing objects, i.e. material ones. Hence instead of saying 
that material objects exist in space and time, we should say that space and time exist vicariously, namely by virtue of the existence (hence change) of material objects. Space and time do not exist independently any more than solidity or motion, life or mind, culture or history. (Cf. Bunge 1977.)

\section{SYSTEM}

A system may be characterized as a complex object whose components are interrelated, as a consequence of which the system behaves in some respects as a unit or whole. Every system can be analyzed into its composition (or set of parts), environment (or set of objects other than the components and related to these), and structure (or set of relations, in particular connections and actions, among the components and these and environmental items). It follows from the above definition of a system, jointly with Postulate 1 and Definition 3 that, if a system is composed of material (real) objects, then it is real itself. More precisely, we derive

Theorem 1. A system is real (material) if, and only if, it is composed exclusively of real (material) parts.

This statement may appear to be trivial but it is not. For one thing it tells us that systems other than physical or chemical systems, such as organisms and societies, are material. For another it entails that, at least according to materialism, the „worlds" composed of ideas — such as fictions and theories - are unreal. What are real are the creators of such ideal ,worlds".

Now that we have the notion of a real (material) system, we may as well add the assumption that renders materialism systemic, to wit,

Postulate 2. Every real (material) object is either a system or a component of a system.

Stated negatively: There are no stray things. The epistemological consequence is obvious: Look for relations, in particular links (or couplings or connections) among things.

Note the following points. First, our version of materialism is dynamicist, for it identifies materiality with changeability. Given the obscurities of dialectics, nothing would be gained and much lost by appending the qualifier 'dialectical'. Second, Postulate 1 should not be mistaken for nominalism (or vulgar materialism, or reism), i.e. the thesis that there are only things, properties being nothing but collections of things, and relations identical with tuples of things. True, we deny the independent existence of properties and relations, but assert instead that things are propertied and related. Third, neither the postulate of materialism nor the accompanying definitions place any restriction on the kind of matter, i.e. on the composition of reality. In particular the above does not involve physicalism, or the thesis that every real object is physical. More on this 
in the next Section. Fourth, Postulate 2, or the hypothesis of systemicity, should not be mistaken for holism. Indeed, holism construes systems as wholes opaque to analysis. On the other hand we conceive of a system as a complex thing with a definite (though changeable) composition, environment, and structure-hence as analyzable.

\section{EMERGENCE}

Materialism is a kind of substance monism: it asserts that there is only one kind of substance, namely matter. (Substance pluralism, on the other hand, holds that there are multiple substances, e.g. matter and mind.) But materialism need not be property monistic, i.e. need not assert that all material objects have a single property, such as spatial extension, or energy, or the ability to join with other objects. Materialism need not even assert that all the properties of material objects are of the same kind, e.g. physical. In particular, Postulate 1 and the accompanying definitions make room for property pluralism as well as the emergence hypothesis and conjectures about the level structure of reality.

Since the notions of emergence and of level are somewhat tricky and suspect in many quarters, we had better start by defining them. To this end we need a prior notion occurring in the definition of the concept of a material system, namely that of composition. The composition of a system is, of course, the set of

\section{its parts. More precisely, the $A$-compositon $\zeta_{A}(x)$ of system $x$, or composition of $x$ on level $A$, is the set of parts, all of kind $A$, of $x$. For example, the atomic composition of a molecule is the set of its atoms; the neuronal composition of a brain is the set of its neurons; and the individual composition of a social system is the set of persons composing it. We are now ready for \\ Definition 5 . Let $x$ be a system with $A$-composition $\zeta_{A}(x)$, and let $P$ be a property of $x$. Then}

(i) $P$ is $A$-resultant (or resultant relative to level $A$ ) if, and only if, $P$ is possessed by every $A$-component of $x$;

(ii) otherwise, i.e. if $P$ is not (possessed by) every $A$-component of $x$, then $P$ is $A$-emergent (or emergent relative to level $A$ ).

For example, the components of a cell are not alive: life is emergent, not resultant, relative to the components of a cell. And perception, feeling, and ideation are functions (activities) of multicellular neuronal systems, that no individual neuron can discharge: they too are emergent. On the other hand mass and energy are resultant properties.

There is nothing mysterious about emergence if conceived of in the above ontological sense. Emergence does become mysterious only when it is characterized epistemologically, namely as whatever property of a system cannot be explained from the components and their relations. But such 
characterization is incorrect, for one must then be able to state both the thesis of the explainability and that of the essential irrationality of emergence. (Needless to say I adopt only the former. But I shall not discuss it here because it is an epistemological thesis, not an ontological one.)

And now a hypothesis about emergence:

Postulate 3. Every system possesses at least one emergent property.

In a sense this hypothesis is trivial, for every system has a composition and a structure, which differ from those of its components. However, the postulate is useful for it draws one's attention to emergence, a much misunderstood and maligned property and one whose recognition allows one to partition the family of materialist ontologies into two subsets. One is the class of ontologies that may be called emergent materialism, because they acknowledge emergence (e.g. R. W. Sellars 1970). Its complement is physicalism (or mechanism or reductive materialism), or the class of ontologies asserting that, whether on the surface or ,at bottom” (or ,,in the last analysis”), every existent is physical (e.g. Smart 1963).

\section{LEVELS AND EVOLUTION}

The emergence postulate suggests looking for emergence mechanisms, such as the clumping of like entities and the merger of unlike ones, as well as for developmental and evolutionary processes in the course of which systems of new species appear. At least the following comprehensive kinds or levels of entity may be distinguished:

Physical level $=$ The set of all physical things

Chemical level $=$ The set of all chemical systems (wherein chemical reactions occur)

Biological level $=$ The set of all cells or multicellular organisms

Social level $=$ The set of all social systems.

We cannot dwell on this taxonomy. Suffice it to note the following points. First, the components of every system belonging to a level above the physical one belong to lower levels. (Actually this relation serves to define the concept of a level in a rigorous manner: see Bunge 1979.) Second, as we climb up the levels pyramid we gain some (emergent) properties but lose others. For example, the social level is composed of animals but is not an organism itself.

Finally we lay down a developmental hypothesis:

Postulate 4. The systems on every level have emerged in the course of some process of assembly of lower level entities.

Postulates 3 and 4 entail

Theorem 2 . Every assembly process is accompanied by the emergence of at least one property. 
There is of course an enormous variety of assembly processes, from mere clumping to the merger of social systems, and there may be entirely new types of assembly processes in store. Moreover, while some of them have been natural others are manmade: these are the cases of human social systems and artifacts (including living beings selected by man and, in the near future, synthesized).

In addition to such developmental assembly processes we make room for evolutionary processes, i.e. unique processes along which absolutely new things emerge, i.e. things possessing properties that no thing has possessed before. In biological evolution such novelties derive from mutation and adaptation; in cultural evolution, from behavior and ideation. Much more could be said about evolution on different levels, but we must close now by adding just one more assumption:

Postulate 5. Some processes are evolutionary.

Again, this postulate is far from trivial: creationism holds that all novelty is a gift of some deity, and physicalism (or mechanistic materialism) maintains that there is never novelty but merely rearrangement of pre-existing units. The above postulate is distinctive of evolutionary materialism.

\section{CONCLUSIONS}

The above definitions, postulates, and theorems constitute the core of a materialist ontology with the following characteristics:

(a) exact: every concept is exact or exactifiable;

(b) systematic: every hypothesis belongs to a hypothetico-deductive system;

(c) scientific: every hypothesis is consistent with contemporary science;

(d) dynamicist: every entity is changeable;

(e) systemist: every entity is a system or a component of such;

(f) emergentist: every system has properties absent from its components; (g) evolutionist: every emergence is a stage in some evolutionary process. So much for a sketch of the new scientific materialism. (For details see Bunge 1977, 1979, 1980, 1981.) To be sure there is some overlap between it and alternative doctrines - otherwise they would not deserve the family name 'materialism'. However, all other materialist ontologies lack at least one of the characteristics listed above. In particular most of the alternatives are inexact (metaphorical and verbal rather than literal and mathematical), unsystematic, dogmatic (unchanging and therefore dated) rather than scientific, or physicalist (mechanistic, reductionistic) rather than emergentist.

\section{REFERENCES}

Bunge, M. Ed. (1972), Exact Philosophy, Dordrecht: Reidel. 
Bunge, M. (1977), The Furniture of the World, Dordrecht-Boston: Reidel.

Bunge, M. (1979), A World of Systems, Dordrecht—Boston: Reidel.

Bunge, M. (1980), The Mind-Body Problem, Oxford-New York: Pergamon.

Bunge, M. (1981), Scientific Materialism, Boston: Reidel.

Coraman, J. W. (1971), Materialism and Sensations, New Haven-London: Yale U. P.

Sellars, R. W. (1970), Principles of Emergent Realism, W. Preston Warren, Ed. St. Louis, Mo. Warren H. Green.

Smart, J. J. C. (1963), Philosophy and Scientific Realism, London: Routledge \& Kegan Paul. 